\title{
VII. On the damping of long waves in a rectangular trough
}

\section{Robert A. Houstoun Ph.D. D.Sc.}

To cite this article: Robert A. Houstoun Ph.D. D.Sc. (1909) VII. On the damping of long waves in a rectangular trough , Philosophical Magazine Series 6, 17:97, 154-164, DOI: $10.1080 / 14786440108636584$

To link to this article: http://dx.doi.org/10.1080/14786440108636584

曲 Published online: 21 Apr 2009.

Submit your article to this journal $\lceil\pi$

Џ Article views: 2

Q View related articles $\asymp$ 


\section{$\left[\begin{array}{ll}154 & ]\end{array}\right.$}

VII. On the Damping of Long Waves in a Rectangular Trough. By RoberT A. Houstoun, Ph.D., D.Sc., Lecturer on Physical Optics in the University of Glasgow*".

$\mathrm{N}$ this paper an expression is derived for the rate of 1 damping of stationary long waves in a rectangular trough, and the result is compared with the results of experiment.

$\S 1$. We shall begin by obtaining the differential equation for long waves, taking viscosity into consideration. In the ordinary treatment of long waves without friction ( $c f$. Lamb's ' Hydrodynamics,' p. 239) the horizontal velocity is the same at all points in the same vertical ; the bottom is perfectly smooth. We shall suppose that there is no slipping at the bottom and that the velocity there is zero.

Let the motion be in one horizontal dimension in a medium of uniform depth $h$. Take the axis of $x$ horizontal and in the direction of motion and the axis of $z$ vertically upwards. Let the bottom of the medium be given by $z=0$, and the surface in its undisturbed state be given by $z=h$.

The ordinate of the free surface corresponding to the abscissa $x$ at time $t$ will be denoted by $h+\zeta$. Then, on the usual assumption that the vertical acceleration of the fluid particles may be neglected, $p$ the pressure at any point $(x, y, z)$ is given by

$$
p=p_{0}+g \rho(h+\zeta-z), . . . .
$$

where $p_{0}$ is the external pressure and $\rho$ the density of the medium. Hence

$$
\frac{\partial^{2} p}{\partial t \partial x}=g \rho \frac{\partial^{2} \zeta}{\partial t \partial x} . . . . . . .
$$

To obtain the equation of motion, consider the element. $\delta x \delta y \delta z$. The difference of the pressure-tbrusts on the ends is

$$
-\frac{\partial p}{\partial x} \delta x \delta y \delta z
$$

The difference of the tangential stresses on the upper and lower faces is

$$
\mu \frac{\partial^{2} u}{\partial z^{2}} \delta x \delta y \delta z
$$

and on the sides is

$$
\mu \frac{\partial^{2} u}{\partial y^{2}} \delta x \delta y \delta z .
$$

* Communicated by Professor A. Gray, F.R.S. 
Damping of Long Waves in a Rectangular Trough. 155

Equating the sum of these terms to the mass-acceleration, we get

$$
\rho \frac{\partial u}{\partial t}=-\frac{\partial p}{\partial t}+\mu\left(\frac{\partial^{2} u}{\partial y^{2}}+\frac{\partial^{2} u}{\partial z^{2}}\right) . \cdot \cdot .
$$

To obtain the equation of continuity, consider the rate at which fluid is entering the space bounded by $x, x+\delta x$, and equate it to the rate at which the fluid in this space is increasing. Hence

$$
\int_{0}^{h}-\frac{\partial u}{\partial x} d z=\frac{\partial \zeta}{\partial t} . . . . . .
$$

Differentiating (4) with respect to $x$ and combining with (2), we obtain

$$
\frac{\partial^{2} p}{\partial t \partial t}=-g \rho \int_{0}^{h} \frac{\partial^{2} u}{\partial x^{2}} d z . . . . . .
$$

Differentiating (3) with respect to $t$ and substituting from (5), we obtain the final equation, writing $p$ for $\mu / \rho$,

$$
\frac{\partial^{2} u}{\partial t^{2}}-g \int_{0}^{h} \frac{\partial^{2} u}{\partial x^{2}} d z-v \frac{\partial}{\partial t}\left(\frac{\partial^{2} u}{\partial y^{2}}+\frac{\partial^{2} u}{\partial z^{2}}\right)=0
$$

This of course reduces to the ordinary equation for long waves if $\nu=0$ and we assume $\partial^{2} u / \partial x^{2}$ to be taken as independent of $z$.

$\S 2$. Let us now apply equation (6) to the case of water contained in a rectangular trough, the ends of which are given by $x=0, x=a$, the sides by $y=0, y=b$, and the bottom by $z=0$. Then, in order that we may apply (6), $\zeta$ and $\partial \zeta / \partial t$ must initially be functions of $x$ alone. Let the initial conditions be

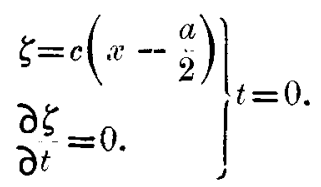

The boundary conditions are

$$
u=0 \text { for } x=0, \quad x=a, \quad y=0, \quad y=b, \quad z=0,
$$

and

$$
\partial u / \partial z=0 \text { for } z=h \text {, }
$$

the latter condition expressing the fact that there is no tangential stress on the free surface. 
As a solution we assume

$$
u=e^{i \sigma t} \mathrm{Z} \sin m \cdot c \sin n y,
$$

where. $Z$ is a function of $z$ alone. Substitution in (6) gives

$$
\left(i \sigma \nu n^{2}-\sigma^{2}\right) Z+g m^{2} \int_{0}^{h} Z d z-i \sigma \nu \frac{\partial^{2} Z}{\partial z^{2}}=0 . .
$$

$\mathrm{Z}$ must be zero when $z=0$ and $\partial Z / \partial z$ must be zero when $z=h$. The solution satisfying these conditions is

$$
Z=\cosh h h-\cdots \cosh h(h-z) . \text {. . . . }
$$

On substituting (8) in ( 7 ) we find that

and

$$
\sigma^{2}-v i \sigma n^{2}+v i \sigma k^{2}=0 \text {. }
$$

$$
\left(\sigma^{2}-g h m^{2}\right)-v i \sigma n^{2}+\frac{g m^{2}}{k} \tanh l h=0 . . \quad . \quad(10
$$

In order to satisfy the boundary conditions we must put $m=q \pi / a$ and $n=r \pi / b$, where $q$ and $r$ are positive integers. From (9) and (10) it is obvious that both $k$ and $\sigma$ are complex. Put $\sigma=p+i \kappa ; \kappa$ is then the reciprocal of the modulus of decay

The complete value of $n$ is given by

$u=$ real part $\Sigma \mathrm{B}_{q r} e^{-\kappa t} \sin p t \sin \frac{q \pi x}{a} \sin \frac{r \pi ! /}{b}[\cosh k h-\cosh k(h-z)]$.

We shall now investigate the rate of decay of the different terms of the above series. Reserving the justification until later, we shall assume that the term vion $n^{2}$ may be neglected in equations (9) and (10) and that tanh $k / h$ may be put equal to unity.

In place of (9) and (10) we have then

$$
\begin{gathered}
k^{2}=\frac{i \sigma}{\nu}, \quad . \quad . \\
\left(\sigma^{2}-g h m^{2}\right)+\frac{.}{k}=0 . \quad . \quad . \quad .
\end{gathered}
$$

and

If $p+i \kappa$ be substituted for $\sigma$ in the above equations, and $\kappa^{2}$ be neglected in comparison with $p^{2}$, we find that $\kappa$ is given by the equation

$$
\kappa^{4}-\stackrel{\nu}{4} h^{2} \kappa^{3}-\left(\frac{\nu p}{\delta / l^{2}}\right)^{2}=0 . \quad \cdot \quad \cdot \quad . \quad .
$$

It is easy to show that this equation has always one, and never more than one, real positive root. This positive root gives the rate of damping, since that rate must be positive; its reciprocal is the modulus of decay. 
If we make the additional assumption that $\sigma / \nu$ is large the expression for $\kappa$ becomes very much simpler. Then (12) may be written

$$
k=\sqrt{\frac{p}{2 v}}(1+i)
$$

Substituting in (13)

$$
p^{2}+2 i p \kappa-g h m^{2}+g m^{2} \sqrt{\overline{2_{p}}}(1-i)=0, \quad .
$$

and equating the imaginary part to zero, we find

$$
\kappa=\frac{g m^{2}}{p} \sqrt{\frac{\nu}{s p}} .
$$

To a first approximation it may be assumed that the viscosity has no effect on the period; then $p / m=\sqrt{g h}$ and the wave-length $\lambda=2 \pi / \mathrm{m}$. Hence we may write

$$
\kappa=\left(\frac{\nu p}{8 h^{2}}\right)^{\frac{1}{2}}=\left(\frac{\nu \pi}{4 \lambda}\right)^{\frac{2}{2}}\left(\frac{g}{h^{3}}\right)^{\frac{1}{4}} \ldots . . .
$$

To find the effect of the viscosity on the period we equate. the real part of equation (15) to zero. Then

$$
p^{2}-g h m^{2}+g m^{2} \sqrt{\frac{\nu}{2 p}}=0
$$

By substitution from (16), this becomes

$$
p^{2}-g h m^{2}+2 p \kappa=0 \text {. }
$$

Hence when $\kappa^{2}$ is neglected in comparison with $g h \mathrm{~m}^{2}$

$$
p=-\kappa+m \sqrt{g h} \text {. }
$$

The period $\mathrm{T}=2 \pi / p . \quad \mathrm{T}_{0}$, the value which we obtain when viscosity is neglected, is $2 \pi /(m \sqrt{g h})$. Hence substituting in (17)

$$
\mathrm{T}=\mathrm{T}_{0}+\frac{\kappa}{2 \pi} \mathrm{T}_{0}{ }^{2} \ldots \text {. . . . . }
$$

We shall next determine the values of the coefficients in the expression for $u$.

* I found, after deriving formula (16), that it had already been obtained by $\mathrm{S}$. Hough for progressive waves in an unlimited medium by a longer method (Proc. Lond. Math. Soc. xxviii. p. 276, 1897); he finds a general expression for the damping of waves in one horizontal dimension, and as a special case gives the formula for long waves. 
Differentiating (11) with respect to $x$ and integrating with respect to $z$ from 0 to $h$, neglecting the term divided by $k$, we find

$$
\int_{0}^{h} \frac{\partial u}{\partial x} d z=\Sigma \mathrm{B}_{q r} \frac{q \pi l}{a} e^{-\kappa t} \sin p t \cos \frac{q \pi x}{a} \sin \frac{r \pi y}{b} \cosh k h .
$$

Integrating this with regard to $t$ and at the same time neglecting the variation of $e^{-\kappa t}$ with $t$, we obtain by means of (4)

$$
\zeta=\Sigma \mathrm{B}_{q r} \frac{q \pi h}{p a} e^{-\kappa t} \cos p t \cos \frac{q \pi x}{a} \sin \frac{r \pi y}{b} \cosh k h . .
$$

Writing

$$
\mathrm{B}_{q r} \frac{q \pi h}{p a} \cosh k h=\mathrm{A}_{q r}
$$

and expressing the initial condition, we obtain

$$
c\left(x-\frac{a}{2}\right)=\Sigma \mathrm{A}_{q r} \cos \frac{q \pi x}{a} \sin \frac{{ }^{r} \pi y}{b} .
$$

If we calculate the different values of $A_{q r}$, we find that the most important ones are as follows :-

$$
\begin{array}{lll}
A_{11}=-\frac{16 a c}{\pi^{3}}, & A_{31}=-\frac{16 a c}{3^{2} \pi^{3}}, & A_{51}=-\frac{16 a c}{5^{2} \pi^{3}}, \\
A_{13}=-\frac{16 a c}{3 \pi^{3}}, & A_{33}=-\frac{16 a c}{3.3^{2} \pi^{3}}, & A_{53}=-\frac{16 a c}{3.5^{2}} \frac{\pi \pi^{3}}{} \\
A_{15}=-\frac{16 a c}{5 \pi^{3}}, & A_{35}=-\frac{16 a c}{5.3^{2} \pi^{3}}, & A_{55}=-\frac{16 a c}{5.5^{2} \pi^{3}}
\end{array}
$$

The values with an even suffix vanish.

For the different terms of (19) $q / p$ is constant and cosh $k h$ increases rapidly with $q$; hence $\mathrm{B}_{q r}$ must converge more rapidly than $\mathrm{A}_{q r}$. For a point near the centre of the trough, the terms for which $q=1$ in the expression for $u$ will be at the very least nine times as great as the terms for which $q=3$. Hence the rate of damping of the vibrations there will be sensibly that of the first term.

\$3. The trough used for the experiments had a slate bottom and plate-glass sides and ends. It was $152 \cdot 4 \mathrm{cms}$. long and $20.3 \mathrm{cms}$. broad inside and the sides were $15.9 \mathrm{cms}$. high. The waves were excited by raising one end of the trough about $1 \mathrm{~cm}$. by means of a lever. Then the lever was held by a catch until the surface was perfectly still. When 
the catch was suddeniy released, the end dropped, the surface of the water was represented for an instant by $\zeta=c\left(x-\frac{a}{2}\right)$ and then began to oscillate about its equilibrium position. A scale was held from above over the surface of the water parallel to the sides of the trough at its middle. Any motes that happened to be in the water were brought to the surface there; the turning-points of one of them were read on the scale as long as the motion lasted, and the rate of damping thus obtained.

The following table gives some results :-

\begin{tabular}{|c|c|c|c|c|c|c|}
\hline$h$. & $\begin{array}{l}\text { Observed } \\
\text { period. }\end{array}$ & $\mathrm{T}_{0}$ & Difference. & $\begin{array}{l}\text { Difference } \\
\frac{x T_{1}^{2}}{2 \pi} \\
\text { caleulated. }\end{array}$ & $\begin{array}{l}x \text { calcu- } \\
\text { lated. }\end{array}$ & $\begin{array}{c}\boldsymbol{x} \\
\text { observed. }\end{array}$ \\
\hline $\begin{array}{r}\text { ems. } \\
1.0\end{array}$ & $\begin{array}{l}\text { secs. } \\
10 \cdot 1\end{array}$ & $\begin{array}{l}\text { secs. } \\
9 \cdot 75\end{array}$ & $\begin{array}{c}\text { secs. } \\
-35\end{array}$ & $\cdot 45$ & $\cdot 028$ & $\cdot 048$ \\
\hline $2 \cdot 0$ & $7 \cdot 12$ & 6.89 & .23 & $\cdot 23$ & .017 & .032 \\
\hline $3 \cdot 0$ & $5 \cdot 80$ & $5 \cdot 62$ & $\cdot 18$ & $\cdot 14$ & .012 & 028 \\
\hline $5 \cdot 0$ & $4 \cdot 49$ & $4 \cdot 35$ & $\cdot 14$ & .09 & $\cdot 0084$ & 023 \\
\hline $7 \cdot 0$ & $3 \cdot 68$ & $3 \cdot 68$ & $\cdot 00$ & .02 & $\cdot 0066$ & .022 \\
\hline 100 & $3 \cdot 12$ & 308 & .04 & $\cdot 01$ & $\cdot 0050$ & .011 \\
\hline
\end{tabular}

The first column gives the depth of the water and the second the observed period. The third gives $\mathrm{T}_{0}$, the theoretical value obtained for the period when viscosity was neglected; the fourth gives the difference of the second and third, and the fifth gives the effect of the viscosity of the water on the period calculated according to (18). The seventh column gives the observed values of $\kappa$, and the sixth gives values of $\kappa$ calculated according to (16). If we use (14) slightly better values are obtained.

The actual value of $\kappa$ is thus much greater than its theoretical value. The difference is due neither to neglecting the otber modes, which die away more rapidly, nor to the vibrations not being small enough, nor to the "residual motion" of the water. The same values were obtained for $\kappa$ when the water had a slight irregular motion in addition to the periodic one. The approximations made in deriving the formula are perfectly justifiable; for, to take a concrete example, for $h=3 \mathrm{cms}$. in equation (10) $\sigma^{2}=2 \cdot 1$ and $\nu \sigma n^{2}=0 \cdot 00033$, and in equation (11) the modulus of $k h$ is about 60 . If we take 
the vertical acceleration into consideration $\boldsymbol{\kappa}$ becomes smaller, instead of greater.

The difference appears to be due to dissipation of energy in the surface-film. All the other conditions being the same, $\kappa$ increases with the time. The following figures taken for $h=5 \mathrm{cms}$. exemplify this :-

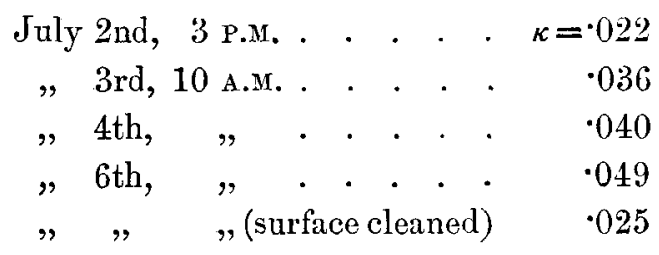

On July 2 nd the trough was cleaned, filled with clean tapwater, and $\kappa$ determined. The water was then left standing in the trongh, and $\kappa$ determined on the $3 \mathrm{rd}, 4$ th, and 6 th. Immediately after the first determination of $\kappa$ on the 6 th the surface was "scraped," the film removed, and $\kappa$ t'ell to half its value.

The values of $\kappa$ determined from a fresh surface were never very consistent; the motes were drawn in different directions owing to the film forming. After the surface had stood some time the values of $\kappa$ were more regular.

Since $\boldsymbol{\kappa}$ increases so much with the time-an increase which we can explain only by the formation of a surface film-it seems reasonable to suppose that the difference between the value of $\kappa$ for a fresh surface and the theoretical value is due to a film which forms with great rapidity. In other words, none of the surfaces were perfectly clean. This view is strengthened by the fact that the addition of minute quantities of lubricating oil or linseed oil to a fresh surface had no appreciable effect on $\kappa$. Apparently there was a film already there.

The impurity seemed to come from the atmosphere of the laboratory and from the putty at the corners of the trough. No improvement was obtained on cleaning the trough with acid or on using distilled water. When a smaller trough, which could he kept clean more easily, was used, the waves were not suitable for accurate measurement.

According to the table the effect of viscosity on the period agrees with its calculated values; the fourth and tifth columns agree. Some other measurements of the effect of viscosity on the period are given below. For these $h$ was calculated from the volume of the water, and the surface was not at all so clean. 
Long Waves in a Rectangular Trough.

\begin{tabular}{|c|c|c|c|c|}
\hline$h$. & $\begin{array}{l}\text { Observed } \\
\text { period. }\end{array}$ & $T_{0}$ & Difference. & $\frac{\kappa \mathrm{T}_{0}^{2}}{\overline{2} \pi}$ \\
\hline $\begin{array}{c}\mathrm{cms} . \\
.5\end{array}$ & $\begin{array}{l}\text { secs. } \\
16 \cdot 7.1\end{array}$ & $\begin{array}{l}\text { secs. } \\
13 \cdot 76\end{array}$ & $\begin{array}{l}\text { secs, } \\
295\end{array}$ & $\begin{array}{l}\text { secs. } \\
1-45\end{array}$ \\
\hline $1 \cdot 0$ & $10 \cdot 57$ & $9 \cdot 75$ & 82 & $\cdot 45$ \\
\hline $2 \cdot 0$ & $7 \cdot 18$ & 6.89 & .29 & .23 \\
\hline $3 \cdot 0$ & $5 \cdot 79$ & $5 \cdot 62$ & $\cdot 17$ & $\cdot 14$ \\
\hline $5 \cdot 0$ & $4 \cdot 425$ & $4 \cdot 35$ & .07 & .09 \\
\hline $7 \cdot 0$ & 370 & $3 \cdot 68$ & .02 & $\cdot 02$ \\
\hline $10 \cdot 0$ & 3.085 & $3 \cdot 08$ & .00 & .01 \\
\hline
\end{tabular}

The agreement is not good, but it ought to be stated that (18) is hardly applicable to such small values of $h$ as 1.0 or 0.5 cins.

If we calculate $\mathrm{T}_{0}$ by the general formula for surface-waves instead of by the formula for long waves, no difference is made, except in the case of $h=10 \mathrm{cms}$. The value for the latter becomes $3 \cdot 10$ secs.

The calming effect of oil on a stormy sea is explained by supposing that the oil floats as a membrane on the surface of the water and hampers its motion by offering resistance to extension. The surface-film here seemed to be easily extensible. To explain the dissipation of energy it is necessary to assume that the membrane opposes the extension with a force proportional to the velocity. The tangential stress on the surface of the water is proportional to and opposed to the velocity there.

We thus have to solve (7) with the conditions

We find

$$
\begin{aligned}
\frac{\partial Z}{\partial z}=-c Z, & z=h, \\
Z=0, & z=0 .
\end{aligned}
$$

$\mathrm{Z}=[\cosh k h-\cosh k(h-z)]-\frac{c}{h}[\sinh k z-\sinh k h+\sinh k(h-z)]$.

Neglecting the term in which $n^{2}$ is a factor, we find that (12) still holds ; but in place of (13) we have

$$
\begin{aligned}
& \left(\sigma^{2}-g h m m^{2}+\frac{2 c}{k^{2}} g m^{2}\right) \\
& \quad+\frac{g m^{2}(1-c h)+c \sigma^{2}}{k} \tanh k h-\frac{2 c g m^{2}}{k^{2}} \frac{1}{\cosh k h}=0 .
\end{aligned}
$$

Phil. Mag. S. 6. Vol. 17. No. 97. Jan. 1909. 
In the coefficient of tanh $k h, \mathrm{gm}^{2} / \mathrm{h}$ may be put equal to $\sigma^{2}$ since the whole term is small. Tanh $k h$ is as usual $=1$, and the $1 / \cosh h k$ term may be neglected as it is very small.

Substituting from (12) in (20) and writing

we get

$$
\sigma=p+i \kappa
$$

$$
p^{2}+i 2 p \kappa-g h m^{2}-i 2 \frac{c v g m^{2}}{p}+g m^{2} \sqrt{\frac{\nu}{2 p}}(1-i)=0 .
$$

The new term is equal to

$$
i 2 p \frac{c v}{h} \text {. }
$$

Hence

$$
\kappa=\left(\frac{\nu p}{8 h^{2}}\right)^{\frac{1}{2}}+\frac{c \nu}{h} . \quad \text {. . . . . . . }
$$

The new surface condition is therefore capable of accounting for the rate of damping. It does not affect the period.

For $h=5 \mathrm{cms}$. and a fresh surface $c$ is about $7, i$.e., for unit velocity the retarding force/unit area is $\frac{1}{14}$ dyne. We can find the effect of a tangential drag of this magnitude on free waves on deep water very readily by the method of Lamb's 'Hydrodynamics,' 3rd edition, \$331.

It is only necessary to add a new term $-\frac{q^{2}}{14}$ to the dissipation function. Then using the notation of that section wo have instead of equation (8)

Therefore

$$
\frac{d}{d t}\left(\frac{1}{2} \rho k c^{2} \alpha^{2}\right)=-2 \mu k^{3} c^{2} \alpha^{2}-\frac{1}{14} k^{2} c^{2} \alpha^{2}
$$

$$
\alpha=\alpha_{0} e^{-2\left(\nu k^{2}+\frac{k}{28_{p}}\right)^{t}},
$$

i. e.,

$$
\kappa=-\frac{8 \pi^{2} \nu}{\lambda^{2}}-\frac{\pi}{7 \rho \lambda} \text {. }
$$

For $\lambda=100 \mathrm{cms}$, the first term $=8 \cdot 10^{-5}$ and the second $4 \cdot 10^{-3}$; hence there cannot be a tangential drag of this magnitude on free waves on deep water.

The damping of the waves in the trough seems in some measure to be due to the film being attached to the sides. According to the theory, if the width of the trough were diminished by half, $\boldsymbol{\kappa}$ at the centre should not be altered appreciably. I had a wooden partition made which could be 
fitted down the middle of the trough dividing it into two. For a fresh surface, $h=5$ cms., on removing this partition $\kappa$ diminished approximately 10 per cent.

$\S 4$. It is interesting to note the manner in which the velocity falls off at the bottom of the trough.

$Z=$ real part $\{\cosh k h-\cosh k(h-z)\}$,

$$
=\cos \sqrt{\frac{p}{2 \nu}} h \cosh \sqrt{\frac{p}{2 \nu}} h-\cos \sqrt{\frac{p}{2 \nu}}(h-z) \cosh \sqrt{\frac{p}{2 \nu}}(h-z) .
$$

Taking $h=5 \mathrm{cms}$. we find that at different depths $\mathrm{Z}$ is roughly proportional to the following numbers, the value at the surface being taken as unity :-

\begin{tabular}{|c|c|c|}
\hline \multirow{2}{*}{\multicolumn{2}{|c|}{$\begin{array}{c}z \\
0.0^{z} \mathrm{~mm} .\end{array}$}} & $Z$ \\
\hline & & $\cdot 000$ \\
\hline 0.5 & , & -129 \\
\hline $1 \cdot 0$ & $"$ & $\cdot 520$ \\
\hline 1.5 & ", & $\cdot 732$ \\
\hline $2 \cdot 0$ & $"$ & $\cdot 811$ \\
\hline $3 \cdot 0$ &, & 1.03 \\
\hline $4 \cdot 0$ & ," & 1.04 \\
\hline $5 \cdot 0$ &, & $1.0 z$ \\
\hline $6 \cdot 0$ & $"$ & $1 \cdot 01$ \\
\hline $7 \cdot 0$ &, & 1.00 \\
\hline
\end{tabular}

Thus the velocity reaches its surface value $3 \mathrm{~mm}$. from the bottom.

$\S \overline{5}$. The case of a circular sheet with symmetry about the centre can be treated in the same way as the rectangular trough.

If $u$ denote the radial velocity the equation of continuity is

$$
\frac{\partial \zeta}{\partial t}=-\int_{0}^{h} \frac{1}{r} \frac{\partial}{\partial r}(u r) d z . \quad \text {. . . . }
$$

The equation of motion is

$$
\rho \frac{\partial u}{\partial t}=-\frac{\partial p}{\partial r}+\mu \frac{\partial^{2} u}{\partial z^{2}} . \quad . \quad . \quad .
$$

Eliminating $p$ by means of (1) and (23) we get

$$
\frac{\partial^{2} u}{\partial t^{2}}=g \int_{0}^{h} \frac{\partial}{\partial r} \frac{1}{r} \frac{\partial(u r)}{\partial r} d z+\nu \frac{\partial^{3} u}{\partial t} \frac{u}{\partial z^{2}} . \quad \text { M } 2 .
$$


164 Mr. A. Stephenson on the Frequency Ranges of

Multiply by $r$, differentiate with regard to $r$, and divide by $r$, and (25) becomes

$$
\frac{\partial^{2}}{\partial t^{2}} \frac{1}{r} \frac{\partial(u r)}{\partial r}=g \int_{0}^{h} \frac{1}{r} \frac{\partial}{\partial r} r \frac{\partial}{\partial r} \frac{1}{r} \frac{\partial(u r)}{\partial r} d z+\nu \frac{\partial^{s}}{\partial t \partial z^{2}} \frac{1}{r} \frac{\partial(u r)}{\partial r} .
$$

Substitute

$$
\frac{1}{r} \frac{\partial(u r)}{\partial r}=\mathrm{J}_{0}(m r) \mathrm{Z}
$$

where $\mathrm{Z}$ is a function of $z$ alone. Then

$$
\begin{aligned}
\frac{1}{r} \frac{\partial}{\partial r} r \frac{\partial}{\partial r} \frac{1}{r} \frac{\partial(u r)}{\partial r} & =\frac{\partial^{2}}{\partial r^{2}} \frac{1}{r} \frac{\partial(u r)}{\partial r}+\frac{1}{r} \frac{\partial}{\partial r} \frac{1}{r} \frac{\partial(u r)}{\partial r} \\
& =\mathrm{Z}\left(\frac{\partial^{2} \mathrm{~J}_{0}(m r)}{\partial r^{2}}+\frac{1}{r} \frac{\partial \mathrm{J}_{0}(m r)}{\partial r}\right) \\
& =-m^{2} \mathrm{~J}_{0}(m r) \mathrm{Z} .
\end{aligned}
$$

Hence $J_{0}(m r)$ cancels out of equation (26). If we assume a time-factor $e^{i \sigma t}$ we have for $Z$

$$
\sigma^{2} Z-g m^{2} \int_{0}^{h} \mathrm{Z} d z+\nu i \sigma \frac{\partial^{2} \mathrm{Z}}{\partial z^{2}}=0, \ldots .
$$

which is the same as equation (7).

VIII. On the Frequency Ranges of Non-generating Force exerting Cumulative Influence. By ANDREW STEPHENSON*.

1. DERIODIC non-generating force acting on a system in oscillation about a position of stable equilibrium, exeris a cumulative action in intensifying or diminishing the amplitude if its frequency is contained within any one of a number of ranges lying in the vicinity of $2 \mu, 2 \mu / 2 ; 2 \mu / 3 \ldots$, where $\mu$ is the natural trequency of the system $\dagger$.

The limits of the leading ranges have been obtained for small non-generating force. It is our object here to give a general method of finding the ranges in magnitude and position when the force is finite, and in particular to obtain the numerical values of the range limits about the double frequency for various intensities.

* Communicated by the Author.

+ "On a Class of Forced Oscillations," Quart. Journ. of Mathematics, No. $168,1906$. 